\title{
ANÁLISE DA INCONTINÊNCIA URINÁRIA NA QUALIDADE DE VIDA DE IDOSAS FREQUENTADORAS DE UM GRUPO DE CONVIVÊNCIA SOCIAL EM MURIAÉ-MG
}

\author{
Giovana Scoparo Muratori de Oliveira ${ }^{\alpha}$ \\ Nittina Anna Araújo Bianchi Botaro ${ }^{b}$ \\ Clarissana Araújo Botaro ${ }^{c}$ \\ Cristiano Andrade Quintão Coelho Rocha ${ }^{d}$
}

\begin{abstract}
Resumo
Este estudo teve como objetivo analisar o impacto da incontinência urinária (IU) na qualidade de vida (QV) de mulheres idosas que participam de um grupo de convivência na cidade de Muriaé-MG, com 60 anos ou mais. $O$ estudo é do tipo transversal de uma amostra proposital. As participantes da pesquisa foram enquadradas nos critérios de inclusão, e foram entrevistadas individualmente por meio de um questionário King's Health Questionnaire (KHQ), que avalia o impacto da IU na QV de mulheres, que contém oito domínios: percepção geral de saúde, impacto da incontinência, limitações de atividades diárias, limitações físicas e sociais, relacionamentos pessoais, emoções, sono / disposição e de gravidade, cuja pontuação varia de o a 100, sendo que a maior pontuação indica pior qualidade de vida. O mesmo questionário também apresenta questões que avaliam a maneira como os sintomas da incontinência, afetam as atividades diárias, as limitações físicas, as relações socias, dentre outros aspectos. A pesquisa mostrou uma correlação entre os domínios, destacando a percepção geral de saúde, sono/disposição e os sintomas que se destacaram foram noctúria, enurese noturna e aumento da frequência de idas ao banheiro (55\%). Em conclusão, a IU apresenta uma influência moderada sobre a QV de mulheres idosas, demonstrando que o fato de estar em constante contato com outros idosos no grupo de convivência por elas frequentado e participando de outras atividades propostas no mesmo, faz com que as mesmas se sintam bem.
\end{abstract}

Palavras-Chave: Métodos epidemiológicos; Questionário; Qualidade de vida e incontinência urinária.

a. Graduada em Fisioterapia pela Faculdade de Minas-FAMINAS, Campus Muriaé-MG. giovanamuratori@hotmail.com

b. Mestranda do Programa de Pós Graduação em História da Universidade Federal de Juiz de Fora - UFJF/MG. nittina.bianchi@hotmail.com

c. Mestre em Ciências da Saúde e Ambiente pelo Centro Universitário Plínio Leite-UNIPLI. Professora Adjunta do Curso de Fisioterapia da Faculdade de Minas-FAMINAS, Campus Muriaé/MG. clafisio@gmail.com.br

d. Mestre em Ciências da Motricidade Humana pela Universidade Castelo Branco - UCB/RJ. Professor Adjunto do Curso de Fisioterapia da Faculdade de Minas-FAMINAS, Campus Muriaé/MG. caqcr@bol.com.br 


\title{
ANALYSIS OF URINARY INCONTINENCE ON QUALITY OH LIFE IN OLDER OFTEN A GROUP OF SOCIAL INTERACTION IN MURIAÉ-MG
}

\begin{abstract}
This study aimed to analyze the impact of urinary incontinence (UI) in the quality of life (QOL) of elder women who are 60 years old or more and are participating in a support group in Muriaé city, Minas Gerais. The research is a study of a purposive sample. The participants were grouped in the inclusion criteria and besides they were interviewed individually and our support to do it was a questionnaire King's Health Questionnaire (KHQ), which assesses the impact of $\mathrm{UI}$ on the QOL of women and contains eight domains: general health perception, incontinence impact, limitations of daily activities, physical and social limitations, personal relationships, emotions, sleep / mood and severity. In addition, there is a ranking from o to 100 in which higher scores indicating worse quality of life. The same questionnaire also poses questions that assess symptoms of incontinence, affecting daily activities, physical limitations, socias relations, among other aspects. The survey showed a correlation between the domains, highlighting the general health perception, sleep / mood and symptoms that stood out were nocturia, nocturnal enuresis and frequent use of the toilet (55\%). In conclusion, was proven that UI has a moderate influence on QOL women, showing that the fact of being in constant contact with other seniors in the living group attended by them and participating in other activities proposed in it, makes the same if feel good.
\end{abstract}

Keywords: Epidemiologic Methods; Questionnaire; Quality of life and urinary incontinence.

\section{INTRODUÇÃO}

Em vários países do Terceiro Mundo, inclusive o Brasil, entre os anos 30 e 60 do século XX, houve um pequeno declínio da mortalidade, com a fecundidade mantendo-se em níveis elevados. Dado o padrão de queda da mortalidade, não houve impacto significativo na estrutura etária proporcional, somente sobre o ritmo de crescimento populacional, fenômeno conhecido, na época, como explosão demográfica.(1)

Ao começar o declínio sustentado da fecundidade é que se dá início ao processo de envelhecimento da uma população. Em vários países que tinham uma população extremamente jovem, quase estável, com o declínio da fecundidade, o ritmo de crescimento anual do número de nascimentos passou imediatamente a cair. ${ }^{(2)} \mathrm{O}$ aumento da expectativa de vida feminina nos últimos anos despertou maior preocupação e interesse com a saúde e qualidade de vida das mulheres. ${ }^{(3)}$
O processo de envelhecimento, embora fisiológico, é permeado por maior vulnerabilidade às doenças (diabetes mellitus, insuficiência cardíaca, entre outras), as quais podem interferir na autonomia, na mobilidade, na destreza manual, na lucidez e na capacidade funcional das vias urinárias inferiores e da bexiga, favorecendo a Incontinência Urinária (IU). Segundo a Sociedade Internacional de Continência, a mesma é definida como a condição na qual a perda involuntária de urina é um problema social ou higiênico e é objetivamente demonstrada, sendo muito comum entre idosos, sua real prevalência é imprecisa, provavelmente devido às diferentes amostras e tipos de IU estudados. ${ }^{(4,5)}$

O termo e o conceito de Qualidade de Vida (QV) surgiram com o crescimento e o desenvolvimento econômico ocorridos após a Segunda Guerra Mundial, nos Estados Unidos da América, para 
descrever o efeito de aquisição de diferentes bens na vida das pessoas. O conceito é subjetivo e, portanto, sua definição é variável. Está relacionado à percepção do indivíduo sobre seu estado de saúde em grandes domínios ou dimensões de sua vida. ${ }^{(6)}$

$\mathrm{A} Q \mathrm{Q}$ está relacionada à autoestima e ao bem-estar pessoal envolvendo aspectos como a capacidade funcional, o nível socioeconômico, o estado emocional, a atividade intelectual, os valores culturais, éticos e a religiosidade e a própria saúde, o ambiente onde vivem, as atividades e o estilo de vida cotidiano. ${ }^{(7)}$

A QV das mulheres incontinentes é afetada de diversas maneiras. Geralmente, as pacientes com IU apresentam idade mais avançada, por vezes durante o climatério. Evidências sugerem que tais alterações fisiológicas parecem desencadear quadros de depressão e ansiedade, intensificados pelas sensações de baixa auto-estima e insatisfação sexual, culminando com a auto-exclusão do convívio social. Passam a se preocupar com a disponibilidade de banheiro, envergonham-se com o odor de urina e sentem-se frequentemente sujas, chegando a apresentar lesões cutâneas como dermatite amoniacal e infecções urinárias repetidas. Muitas apresentam dificuldade no intercurso sexual, seja por perda de urina, pelo modo de interrompê-lo para urinar ou simplesmente por vergonha perante o parceiro. ${ }^{(8)}$

O presente trabalho propôs pesquisar a correlação da presença de IU e QV em idosas que frequentam um grupo de convivência no município de Muriaé - MG. Diante das diversas alterações sociais, pessoais e emocionais de idosas frente à perda urinária, é relevante avaliar a QV das mesmas, valorizando assim a opinião da paciente sobre sua própria condição de saúde.

\section{METODOLOGIA}

O presente estudo é do tipo transversal de caráter exploratório e descritivo. Realizado em idosas numa amostra intencional num grupo de convivência do município de Muriaé - MG, onde o pesquisador usa o julgamento para selecionar os membros da população que são boas fontes de informação precisa. A pesquisa foi realizado no período de maio de 2010 ,

A amostra foi composta por 20 mulheres idosas com 60 anos de idade ou mais, ativas, entrevistadas com queixa de IU, participantes de um grupo de convivência social e que aceitaram ser voluntárias na participação no estudo. A idade média foi de 73,55 $( \pm 6,65)$ anos. Assinaram o Termo de Consentimento Livre e Esclarecido de acordo com a resolução 196/96 do Conselho Nacional de Saúde (2000), para que assim todas as informações pertinentes às pessoas em questão pudessem ser preservadas no processo de análise. $\mathrm{O}$ estudo obteve parecer favorável do Comitê de Ética e Pesquisa, da Faculdade de Minas - FAMINAS, Muriaé.

As idosas foram avaliadas individualmente por meio do questionário King's Health Questionnaire (KHQ) de qualidade de vida na IU da mulher, sendo realizado na forma de entrevista.

O KHQ é um instrumento específico da avaliação da QV de pessoas com IU, abordando seu impacto nos diferentes domínios da QV, assim como sintomas por ele percebidos. É composto por trinta perguntas, sendo organizadas em nove domínios. Relatando respectivamente, a percepção da saúde, impacto da incontinência, limitações das tarefas, limitação física, limitação social, relacionamento pessoal, emoções, sono/disposição e medidas de gravidade. Existe também uma escala de sintomas que é composta pelos seguintes itens: frequência urinária, noctúria, urgência, hiperreflexia vesical, IUE, enurese noturna, incontinência no intercurso sexual, infecções urinárias e dor na bexiga. ${ }^{(6)}$

A todas as respostas são atribuídos valores numéricos, somados e avaliados por domínio. Os valores são, então, calculados por meio de fórmula matemática, obtendo-se assim, o escore da QV que varia de o a 100 pontos, considerando-se que quanto maior o número obtido, pior a QV.

De acordo com as características do estudo, sugeriu-se o uso da estatística descritiva (caracteri- 
zação do universo amostral) utilizando medida de tendência central (média) e medida de dispersão (desvio padrão).

\section{RESULTADOS}

$\mathrm{Na}$ Tabela 1 são apresentadas a caracterização da amostra, como o número de participantes, a média de idade e desvio padrão.

Tabela 1. Caracterização da amostra

\begin{tabular}{c|c|c}
\hline VARIÁVEIS & MÉdIA & DeSVIO PADRÃo \\
\hline Número de participantes (20) & - & - \\
\cline { 2 - 3 } Idade & 73,55 & 6,55 \\
\hline
\end{tabular}

De acordo com o Gráfico 1 abaixo, pode-se perceber que no resultado do domínio 1 (percepção geral de saúde) $40 \%$ das participantes apresentaram classificação mau e $60 \%$ como sendo regular.

Gráfico 1. Percepção geral de saúde

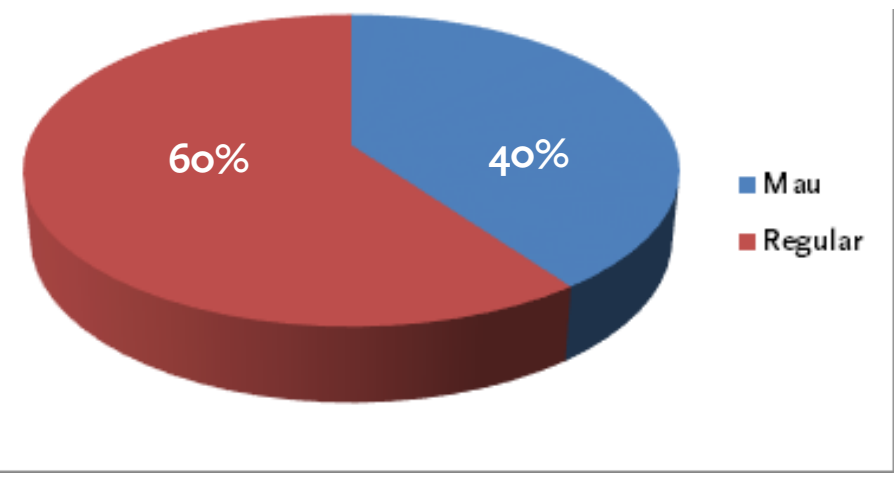

O Gráfico 2 refere-se aos resultados do domínio 2 (impacto da incontinência urinária). Nele pode-se perceber que $25 \%$ das participantes disseram afetar em nada sua vida diária, $45 \%$ um pouco, $25 \%$ moderadamente e $5 \%$ mencionaram que afeta muito.

Gráfico 2. Impacto da incontinência urinária

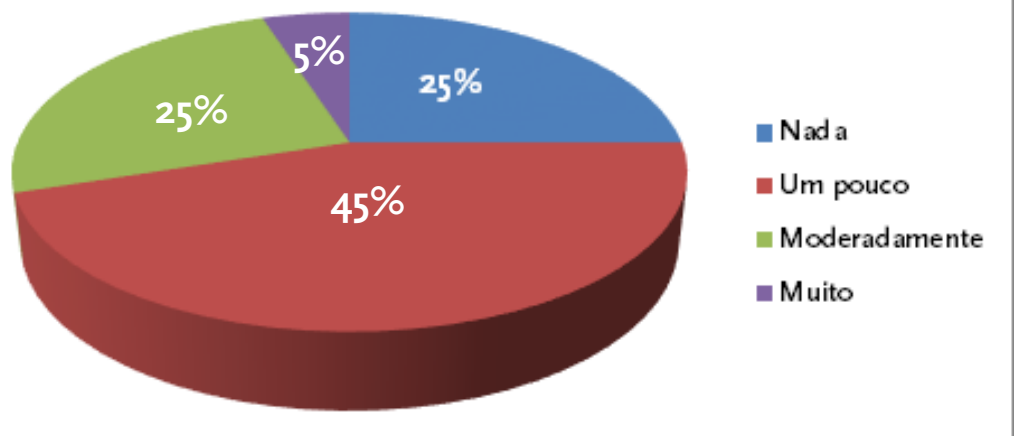


O Gráfico 3 mostra os resultados do domínio 3 (limitações de atividades diárias). Verificou-se que $45 \%$ das participantes informaram que a inconti- nência urinária não afeta em nada suas atividades de vida diária, $35 \%$ informaram que afeta um pouco, $15 \%$ moderadamente e $5 \%$ afeta muito.

Gráfico 3. Limitações de atividades diárias

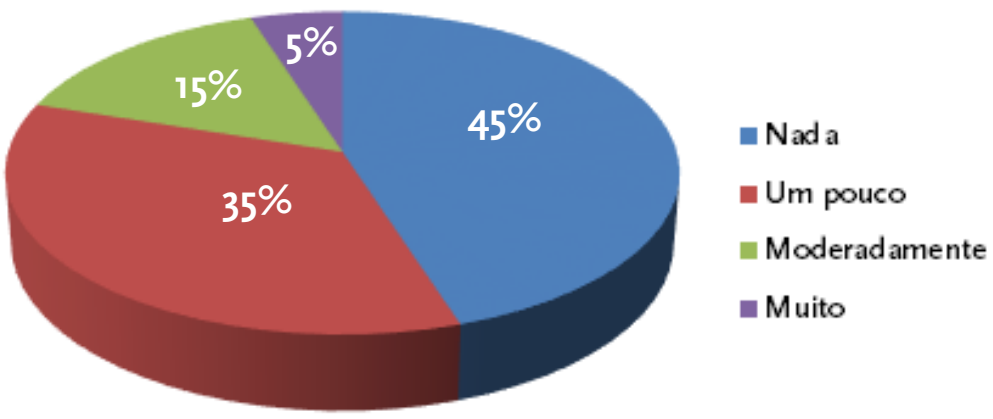

O Gráfico 4 mostra os resultados do domínio 6 (Emoções). Verificou-se que $50 \%$ das participantes informaram que a incontinência urinária não afeta em nada suas emoções, seguido de $35 \%$ um pouco, $5 \%$ moderadamente e $10 \%$ muito.

Gráfico 4. Emoções

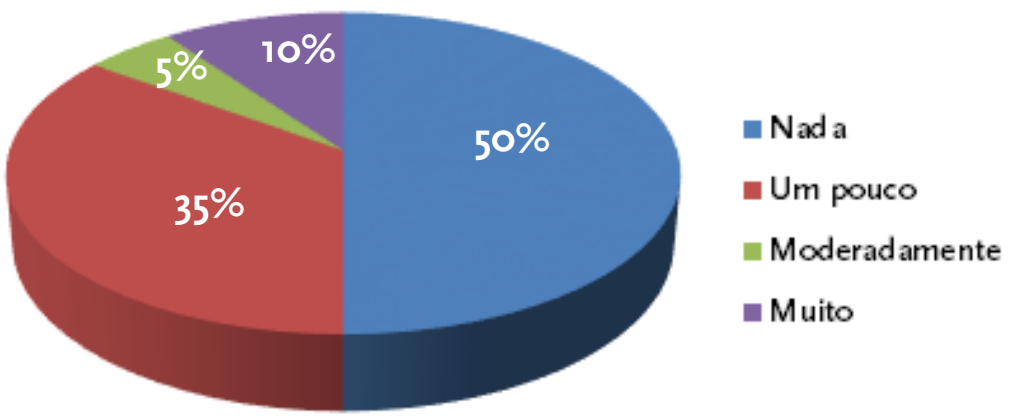

$\mathrm{Na}$ Tabela 2 estão apresentados os valores dos escores da pontuação de cada um dos domínios. Pode-se observar que os domínios que apresentaram escores mais elevados foram respectivamente foram: domínio 1 (percepção geral de saúde) com uma média de 40 pontos, seguido do domínio 7 (sono/disposição) com 37,47 pontos, domínio 2 (impacto da incontinência) com 36,63 pontos e o domínio 4 (limitações físicas) com 27,48 pontos. No entanto os domínios com os escores mais baixos respectivamente foram: domínio 3 (limitações de atividades diárias) com uma média de 23,3 pontos, seguido do domínio 6 (emoções) com 21 pontos, domínio 5 (relações pessoais) com 10,82 pontos e por último o domínio 4 (limitações sociais) com 10,54 pontos. 
Tabela 2 - Valores dos escores obtidos (Média e Desvio padrão), entre os domínios do KHQ, em idosas ativas.

\begin{tabular}{l|c|c}
\hline \multicolumn{1}{c|}{ DoMínIo Do KHQ } & MÉDIA & DESVIO PADRÃo \\
\hline Percepção geral de saúde & 40 & $\pm 12,56$ \\
\hline Impacto da Incontinência & 36,63 & $\pm 28,39$ \\
\hline Limitações de atividades diárias & 23,3 & $\pm 29,8$ \\
\hline Limitações físicas & 27,48 & $\pm 29,24$ \\
\hline Limitações sociais & 10,54 & $\pm 15,47$ \\
\hline Relações pessoais & 10,82 & $\pm 18,92$ \\
\hline Emoções & 21 & $\pm 26,45$ \\
\hline Sono/disposição & 37,47 & $\pm 36,23$ \\
\hline Medidas de gravidade & 17,46 & $\pm 20,56$ \\
\hline
\end{tabular}

Fonte: King's Health Questionnaire.

A Tabela 3 apresenta a distribuição percentual das idosas segundo a interferência dos sintomas urinários na qualidade de vida. Nela pode-se perceberem relação a alguns sintomas que em se tratando de bexiga hiperativa, 90\% das entrevistadas disseram não apresentar nenhum sintoma e somente $10 \%$ relataram ter de forma moderadamente. Relativo ao sintoma dor na bexiga, $80 \%$ não relataram apresentarem nenhum sintoma, $10 \%$ um pouco e $10 \%$ moderadamente. No que se diz respeito ao sintoma noctúria, 20\% disseram haver um pouco, 50\% moderadamente e $30 \%$ muito. Em relação ao sintoma infecções frequentes, $45 \%$ apresentaram ter um pouco, $30 \%$ moderadamente e $25 \%$ sem sintoma.

Tabela 3. Distribuição percentual das idosas segundo a interferência dos sintomas urinários na QV.

\begin{tabular}{|c|c|c|c|c|}
\hline SINTOMAS & $\begin{array}{c}\text { UM POUCO } \\
\text { N\% }\end{array}$ & $\begin{array}{c}\text { MODERADAMENTE } \\
\mathrm{N} \%\end{array}$ & $\begin{array}{l}\text { MuITO } \\
\text { N \% }\end{array}$ & $\begin{array}{c}\text { SEM SINTOMA } \\
\text { N \% }\end{array}$ \\
\hline Frequência & $630 \%$ & $1155 \%$ & $315 \%$ & О ०\% \\
\hline Noctúria & $420 \%$ & $1050 \%$ & $630 \%$ & O O\% \\
\hline Urgência & $210 \%$ & $525 \%$ & $315 \%$ & $1050 \%$ \\
\hline Bexiga Hiperativa & О ०\% & $210 \%$ & O ०\% & $1890 \%$ \\
\hline IUE & $525 \%$ & $315 \%$ & O O\% & $1260 \%$ \\
\hline Enurese Noturna & $1155 \%$ & $525 \%$ & O ०\% & $420 \%$ \\
\hline $\begin{array}{l}\text { Incontinência no } \\
\text { intercurso sexual }\end{array}$ & $420 \%$ & ० ०\% & ० ०\% & $1680 \%$ \\
\hline Infecções frequentes & $945 \%$ & $630 \%$ & О ०\% & $525 \%$ \\
\hline Dor na bexiga & $210 \%$ & $210 \%$ & O O\% & $1680 \%$ \\
\hline
\end{tabular}

IUE: Incontinência urinária de emergência.

Fonte: Dados do pesquisador (2010).

\section{DISCUSSÃO}

Em um estudo, ${ }^{(9)}$ com 456 mulheres no climatério, $35 \%$ apresentaram perda urinária por esforços e de acordo com outro estudo, ${ }^{(10)}$ a IU é uma condição que afeta principalmente mulheres idosas em todo o mundo. No estudo de um grupo, (11) foi verificado que a prevalência de incontinência urinária em 
um grupo de mulheres com idade entre 20 a 80 anos foi de $44,6 \%$ e aqueles que vivem em locais de poucos recursos parecem ser mais afetados. ${ }^{(12)}$ Esses dados corroboram com a pesquisa, visto que o grupo amostral se concentrou na população de idosos, ou seja, com idade a partir 60 anos.

$\mathrm{O}$ conceito de $\mathrm{QV}$ está relacionado à percepção do indivíduo sobre grandes domínios ou dimensões de sua vida, entre eles a auto-percepção sobre seu estado de saúde. ${ }^{(13)}$ Existem evidências de que indivíduos com IU vivenciam transtornos mentais comuns, podendo influenciar em sua QV. ${ }^{(6)}$ No entanto, no presente trabalho, prevaleceu "regular" a percepção geral da saúde das entrevistadas, de acordo com a pontuação do escore apresentado (40 pontos), sendo este o domínio de maior média do atual estudo. No entanto, em outro trabalho realizado, (14) a maior média foi no impacto da incontinência urinária. Alguns autores acreditam que estes resultados podem ser influenciados por uma questão cultural, levando ao idoso acreditar que a IU faz parte do envelhecimento natural. (5)

No domínio que abrange as limitações sociais e as relações pessoais percebe-se os escores mais baixos entre os domínios. Há evidências de que os incontinentes experimentam sentimentos de solidão, tristeza e depressão mais expressivos que os continentes e que a influência na QV varia de acordo com o tipo de incontinência e com a percepção individual do problema. ${ }^{(4)}$ As idosas deste trabalho podem ter experimentado em algum momento, os sentimentos negativos citados acima e com isso, os mesmos terem influenciado a resposta deste domínio em parte da amostra.

Em um estudo avaliando a QV de mulheres incontinentes, ${ }^{(14)}$ encontrou como resultado que $50 \%$ delas disseram "muito" e apenas uma delas relatou que a IU não interfere em sua vida. As divergências nos resultados encontrados podem ser explicadas talvez pelas características específicas da população estudada.

A maior porcentagem no referente domínio limitações de atividades diárias foi o pontos, mostrando que a maioria das idosas entrevistadas não apresen- tam dificuldades para executar suas atividades diárias, representando a quinta melhor média entre os domínios. Esses achados podem ser explicados pela participação da amostra em um grupo de convivência de idosos, onde são realizadas atividades como dança, ginástica laboral, pintura, alfabetização, bingo, os quais estimulam o contato entre os participantes e realização de atividade física.

O exercício físico leva o indivíduo a uma maior participação social, resultando em um bom nível de bem-estar biopsicofísico, fatores esses que contribuem para a melhoria de sua qualidade de vida, proporcionando maior longevidade. ${ }^{(15)}$ Podendo também ser confirmado em outro estudo, (1) onde o grupo que pratica exercício teve um desempenho superior em todos os domínios do questionário aplicado comparado com o grupo controle. Estes resultados são consistentes, e demonstraram que a atividade física é um importante fator para um melhor envelhecimento.

O maior número de idosas incontinentes relatou não apresentar limitação social com relação ao seu problema. No entanto a literatura faz referências à tendência de isolamento ao qual estão sujeitos os incontinentes. ${ }^{(4)} \mathrm{Em}$ outro estudo, ${ }^{(16)}$ foi verificado que a incontinência urinária afeta negativamente a vida de $25-50 \%$ das mulheres idosas, principalmente devido a sentimentos de vergonha, dessa forma limitando em atividades de interações sociais. A IU causa impacto na vida social, provocando restrições quanto à frequentar lugares públicos, viajar, dormir fora de casa e até fazer visitas aos amigos. ${ }^{(17)}$ Estas encontram relacionadas, no questionário, com a atividade sexual, a relação com o companheiro.

Nos anos 6o, terapeutas concluíram que fatores psicológicos eram os responsáveis primários pela perda da função sexual e que deixar de fazer sexo apressaria o envelhecimento. Ainda agora tanto a geriatria como a sexologia continua a proclamar que o sexo é um componente necessário para o sucesso do envelhecimento. ${ }^{(18)}$ No entanto a maior parte, da amostra, não possui relação sexual ou respondeu que a perda de urina interfere um pouco 
$(20 \%)$ no intercurso sexual, podendo influenciar no processo de envelhecimento.

As mulheres vivem, em média, sete anos mais do que os homens e estão vivendo mais do que nunca. ${ }^{(19)}$ Outra característica deste grupo populacional é que existe uma maior proporção de viúvas do que em qualquer outra faixa etária. (20) Podendo ser a explicação para a maior prevalência na resposta "não se aplica" do domínio relações pessoais, mesmo não sendo questionado o estado civil das participantes.

Em um estudo realizado, ${ }^{(5)}$ uma das alterações mais frequentes do ato urinário no idoso é a noctúria, decorrente do aumento na secreção de vasopressina e do hormônio natriurético resultando em distúrbios do sono fazendo com que pessoas com mais de 65 anos apresentem um a dois episódios de noctúria mesmo na ausência de qualquer enfermidade. O mesmo resultado pode ser confirmado por outro estudo, ${ }^{(21)}$ onde os autores afirmam que a qualidade do sono no idoso está associada a sintomas como noctúria e enurese noturna. As medidas de precauções podem ser exemplificadas como, fazer uso de absorvente, controlar o quanto de líquido é ingerido, trocar roupa íntima quando estiver molhada e se preocupar com o cheiro de urina.

$\mathrm{Na}$ pesquisa realizada, ${ }^{(8)}$ a QV das mulheres incontinentes é afetada de diversas maneiras: preocupam-se constantemente com a disponibilidade de banheiros e envergonham-se com o odor de urina, apresentando dificuldade no intercurso sexual, seja pelo medo de interrompê-lo para urinar ou simplesmente por vergonha perante o parceiro. Essas evidências não corroboram com o presente artigo, já que apenas $20 \%$ das idosas disseram haver um pouco de dificuldade no intercurso sexual e $80 \%$ relataram não haver nenhum sintoma nesse quesito.

Em um estudo realizado(22) com o objetivo de avaliar a QV de mulheres após tratamento fisioterapêutico da IU de esforço, observou-se as limitações sociais das incontinentes antes e após o tratamento, obtendo como resultado uma melhora significativa dos escores limitações sociais, emoções e sono/disposição. A Sociedade Internacional de Continência reconhece a associação positiva da intervenção fisioterapêutica e QV em mulheres com perda de urina, indicando a fisioterapia como a opção de primeira linha para IU, devido ao baixo custo e baixo risco e eficácia.

Um estudo realizado na Índia ${ }^{(12)}$ com 598 mulheres na faixa etária de 20 a 60 anos de idade enfatiza que as intervenções para prevenir ou aliviar a UI sobre a saúde das mulheres afetadas é um desafio por causa das atitudes negativas. Por outro lado, é de suma importância que a intervenção fisioterapêutica seja realizada, afim de contribuir para a melhor qualidade de vida de idosas incontinentes. $O$ tratamento do idoso requer o empenho de uma equipe multiprofissional que utiliza uma avaliação abrangente multidimensional, para traçar a forma mais adequada de assistência. ${ }^{(23)}$ Portanto, profissionais de saúde devem concentrar seus esforços em melhorar a consciência deste problema entre as mulheres e assegurar-lhes que isso não é uma condição de humilhação e tabu, proporionando cuidados de saúde apropriados, mudanças de estilo de vida simples e apoio psicossoial. ${ }^{(1)}$

\section{CONCLUSÃO}

A Incontinência Urinária como vista nesta pesquisa, de acordo com a pontuação dos escores dos domínios avaliados, apresentou moderada influência sobre a Qualidade de Vida das idosas entrevistadas, sendo o domínio percepção da saúde de maior média, seguido de sono/disposição, demonstrando que pelo fato de estarem em constante contato com outros idosos, participando de atividades no grupo de convivência frequentado por elas, faz com que as mesmas tenham um maior bem-estar.

Quanto aos sintomas questionados, os que mais se destacaram foram noctúria, enurese noturna, frequência de idas ao banheiro o que incomoda muito as incontinentes. Por outro lado as idosas perceberam que o KHQ é um instrumento de investigação importante de avaliação, devido a sua praticidade e rapidez de aplicação. 
Sendo assim, para o restabelecimento da QV dessas idosas o profissional de saúde, especialmente o fisioterapeuta, deve estar atento e comprometido em melhorar a auto-estima, as relações pessoais, físicas e sociais orientando e intervindo quando necessário.

\section{REFERÊNCIAS}

1. Motta LB et al. Novas competências profissionais em saúde e o envelhecimento populacional brasileiro: integralidade, interdisciplinaridade e intersetorialidade. Ciênc. saúde coletiva. 2007; 12(2).

2. Carvalho JAM, Garcia RA. O envelhecimento da população brasileira: um enfoque demográfico. Cad. saúde pública. 2003; 19(3).

3. Berlezi EM et al. Incontinência urinária em mulheres no período pós-menopausa: um problema de saúde pública. Rev. bras. geriatr. gerontol. 2009; 12(2): 159-73.

4. Abreu NS et al. Qualidade de vida na perspectiva de idosos com incontinência urinária. Rev. bras. fisioter. 2007; 11(6).

5. Reis RB et al. Incontinência urinária no idoso. Acta cir. bras. 2003; 18(5).

6. Fonseca ES et al. Validação do questionário de qualidade de vida (King's Healt Questionnaire) em mulheres brasileiras com incontinência urinária. Rev. bras. ginecol. obstet. 2005; 27(5).

7. Castro JC et al. Níveis de qualidade de vida em idosas ativas praticantes de dança, musculação e meditação. Rev. bras. geriatr. gerontol. 2009; 12(2): 255-265.

8. Auge AP et al. Comparações entre os índices de qualidade de vida em mulheres com incontinência urinárias submetidas ou não ao tratamento cirúrgico. Rev. bras. ginecol. obstet. 2006; 28(6).

9. Guarise T et al. Procura de serviço médico por mulheres com incontinência urinária. Rev. bras. ginecol. obstet.. 2001; 23(7).

10. Lopes MHBM, Higa R. Restrições causadas pela incontinência urinária à vida da mulher. Revista Esc. Enferm. USP. 2006; 4O(1): 34-41.
11. Sensoy N, Dogan N, Ozek B, Karaaslan L. Urinary incontinence in women: prevalence rates, risk factors and impact on quality of life. Pak J Med Sci. 2013; 29(3): 821.

12. Seshan V, Muliira JK. Self-reported urinary incontinence and factors associated with symptom severity in community dwelling adult women: implications for women's health promotion. BMC Women's Health. 2013; 13(16).

13. Honório GJS et al. Análise da qualidade de vida em mulheres com incontinência urinária antes e após tratamento fisioterapêutico. ACM: arq. Catarin. med. 2009; 38(4).

14. Alves CCFS, Rabelo, CSS, Maruoka FY. Impacto da incontinência urinária na qualidade de vida em mulheres de 40 a 70 anos na cidade de Belém-PA, Belém [monografia]. Manaus: Curso de Fisioterapia, Centro de Ciências Biológicas e da Saúde da Universidade da Amazônia; 2009.

15. Cheik NC et al. Efeitos do exercício físico e da atividade física na depressão e ansiedade em indivíduos idosos. Rev Bras Ciência e Movimento. 2003; 11(3).

16. Barentsen JA, Visser E, Hofstetter H, Maris AM, Dekker JH, Bock GH. Severity, not type, is the main predictor of decreased quality of life in elderly women with urinary incontinence: a population-based study as part of a randomized controlled trial in primary care. Health Qual Life Outcomes. 2O12; 10(153).

17. Borges JBR et al. Avaliação da qualidade de vida em mulheres com Incontinência Urinária pelo uso do King's Health Questionnaire. Rev Einstein. 2009; 7(3).

18. Bulcão $C B$ et al. Aspectos fisiológicos, cognitivos e psicossociais da senescência sexual. Rev Ciência e Cognição. 2004; 1(1).

19. Salgado CDS. Mulher idosa: a feminização da velhice. Estudo Interdisciplinar do Envelhecimento. 2002 4(2): 7-19.

2O. Leite MT, Hildebrandt LM, Kirchner RM, Winck MT, Silva LAA, Franco GP. Estado cognitivo e condições de saúde de idosos que participam de grupos de convivência. Rev. gaúch enferm. 2012; 33(4):64-71.

21. Anais do XI Encontro Latino Americano de Iniciação Científica e VII Encontro Latino 
Americano de Pós-Graduação; 2007; São José dos Campos. São José dos Campos: MEC. 2007. $3545 p$

22. Rett MT et al. Qualidade de vida em mulheres após tratamento da incontinência urinária de esforço com fisioterapia. Rev. bras. ginecol. obstet. 2007; 29(3): 134-40.
23. Lopes BL, Gazzola JM, Lemos ND, Ricci NA. Independência funcional e os fatores que influenciam no âmbito de assistência domiciliária ao idoso. Rev. bras. geriatr. gerontol. 2007; 10(3): 2-11.

24. Figueiredo EM et al. Perfil sociodemográfico e clínico de usuárias de serviço de Fisioterapia Uroginecológica da rede pública. Rev. bras. fisioter. 2008; 12(2). 